\title{
ARTICLE
}

\section{Development of a micro imaging system for element-selective analysis by coupling of focused ion beam and resonance ionization mass spectrometry}

\author{
Masato Morita $^{\mathrm{a}}$, Hideki Tomita ${ }^{\mathrm{b}}$, Ryohei Ohtake ${ }^{\mathrm{b}}$, Keita Kanenari ${ }^{\mathrm{a}}$, Atsushi Nakamura ${ }^{\mathrm{b}}$, Kosuke Saito ${ }^{\mathrm{b}}$, \\ Toshihide Kawai ${ }^{\mathrm{c}}$, Takeo Okumura ${ }^{\mathrm{c}}$, Volker Sonnenschein ${ }^{\mathrm{b}}$ and Tetsuo Sakamoto ${ }^{\mathrm{a}^{*}}$ \\ ${ }^{a}$ Department of Applied Physics, Kogakuin University, 2665-1 Nakano-machi, Hachioji-shi, Tokyo-to, 192-0015, Japan; \\ ${ }^{b}$ Department of Quantum Engineering, Nagoya University, Furo-cho, Chikusa-ku, Nagoya-shi, Aichi-ken, 464-8603, Japan; \\ 'Japan Neutron Optics Inc., 20-5, Takeshima-cho, Gamagori-shi, Aichi-ken, 443-0031, Japan
}

\begin{abstract}
Characterizing the chemical and isotopic compositions of radioactive materials is important in determining origins, histories and so on. For the actual situation such as particle analysis, micro imaging with high elemental selectivity is necessary. Resonance laser sputtered neutral mass spectrometry (R-SNMS) is very effective analytical method for micro imaging of radioactive materials. In this study, the new Ti:Sapphire laser was developed for R-SNMS was introduced into TOF-SIMS apparatus. And the micro imaging of resonance ionized ${ }^{238} \mathrm{U}$ from an autunite was obtained.
\end{abstract}

Keywords: SIMS; RIMS; micro imaging; SNMS; uranium

\section{Introduction}

It is an urgent task to clarify the origin, history, and intended use of radioactive materials. Development of characterizing method on the precise chemical and isotope analysis with micro scale is one of the important challenges for both nuclear industries and environmental sciences. In particular, micro scale analysis can accurately reveal individual information even in the mixture and matrix sample such as particle analysis.

Secondary ion mass spectrometry (SIMS) is one of the surface imaging techniques, and is generally suitable for the trace analysis of micro area. In particular, time-of-flight SIMS (TOF-SIMS) has no limitation in mass range, it is suitable for analysis of multi-element and unknown sample. Several mass spectrometric techniques exist for isotopic analysis. The SIMS is effective in analyzing solid samples with simple preparation and has demonstrated excellent precision and sensitivity on uranium oxide reference materials [1]. But it has a problem of isobaric interference which derives from non-ionization-selectivity [2]. In order to eliminate the isobaric interference, it is necessary to improve the mass resolution, but in addition to the increase and complication of the apparatus, there are problems such as trade-off with the sensitivity. Therefore, solving isobaric interference by means of high mass resolution is not practical. Thus, elemental isobaric interferences in the actinide mass range cannot be resolved by SIMS (e.g., ${ }^{238} \mathrm{U} /{ }^{238} \mathrm{Pu}$ ).

Resonance ionization mass spectrometry (RIMS) is

*Corresponding author. Email: ct13087@ns.kogakuin.ac.jp known as element-selective analysis for nuclides. The preparation such as chemical separation and concentration to remove constituents that may cause isobaric interference is not required. Other elements are transparent at the wavelengths specific to the element of interest and thus remain as undetected neutrals.

In order to analyze the dynamics of radioactive materials, we combined these two techniques (which is called "resonant laser sputtered neutral mass spectrometry (R-SNMS)") and is aiming at micro area imaging of specific nuclides. In the R-SNMS, the sputtered neutrals from solid sample surface by ion beam bombardment are resonantly-ionized with irradiated pulsed laser passing through near the sample surface. Figure 1 shows the schematic of the principle of R-SNMS. In this study, high repetition rate, high power and tunable-laser based on Ti:Sapphire (Ti:Sa) laser was developed for R-SNMS, and introduced into TOF-SIMS.

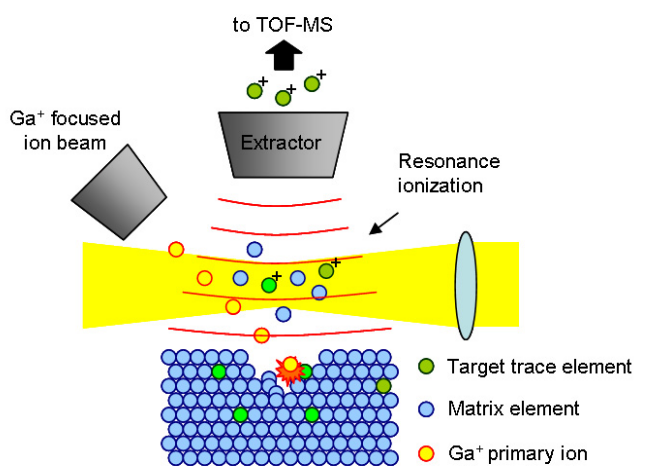

Figure 1. Schematic of resonant laser sputtered neutral mass spectrometry. 


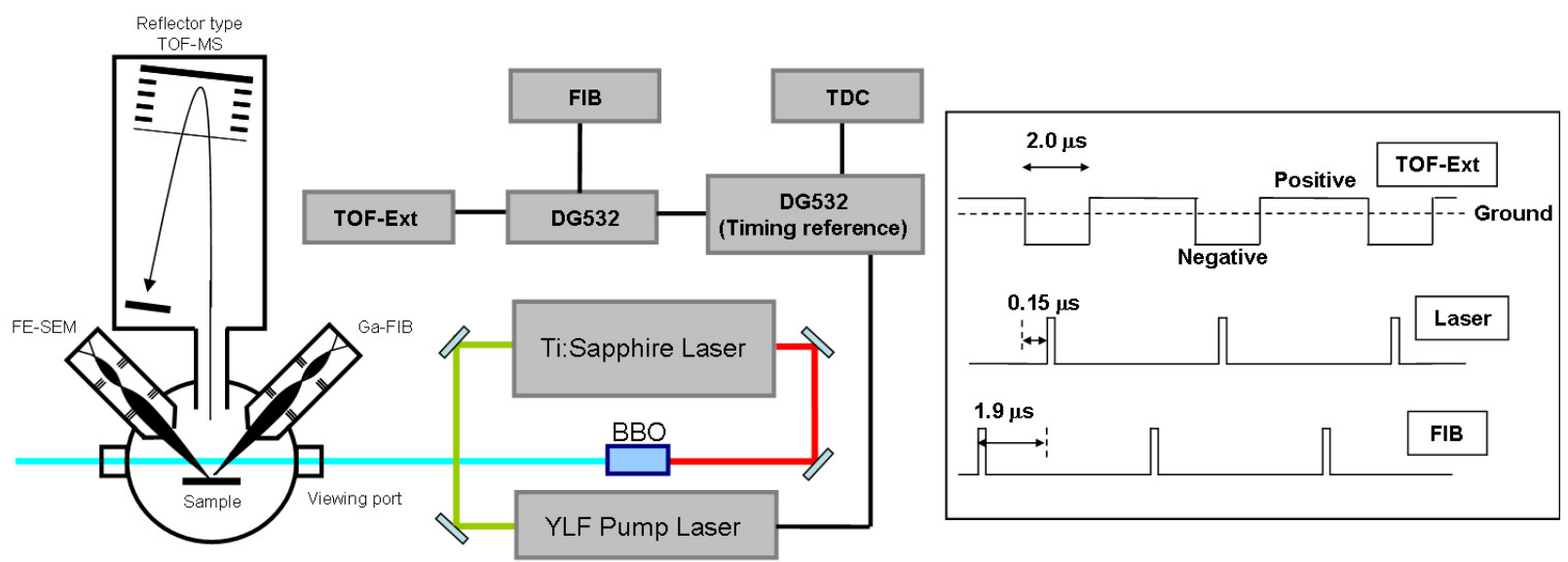

Figure 2. Experimental setup of the laser system for resonant laser sputtered neutral mass spectrometry, and time sequence of triggers for FIB, laser and TOF-Extractor.

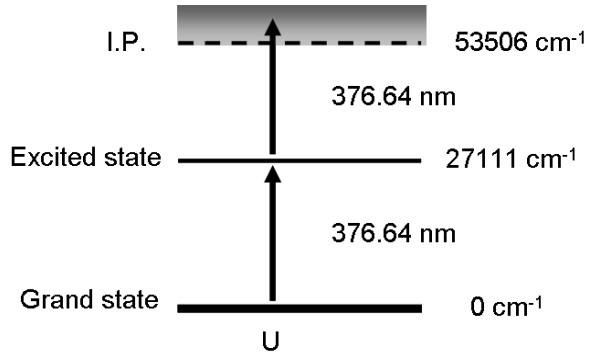

Figure 3. The one-color, two-photon, resonance ionization scheme used in this work.

\section{Experimental}

\subsection{Resonant Laser SNMS System}

Figure 2 shows the experimental setup of R-SNMS in this study. It consisted of a TOF-SIMS apparatus, which was modified for resonant laser post-ionization with a new Ti:Sa laser system. The TOF-SIMS used in the study was developed by us, which already was equipped with non-resonant laser (NR-)SNMS measurement system $[3,4]$. The TOF-SIMS used in this study was consisted from a gallium focused ion beam (FIB), a field emission electron beam, a main vacuum chamber and a reflector-type TOF mass spectrometer. The base pressure was around $5 \times 10^{-6} \mathrm{~Pa}$. The FIB was operated at $30 \mathrm{keV}$ energy, and its pulse width can be varied from 20 to $5000 \mathrm{~ns}$. Beam current and spot diameter are adjusted by using size-selectable apertures located between the two electrostatic lenses other than the selection of upper or lower choppers. The maximum lateral resolution is about $40 \mathrm{~nm}$. Element selected images can be obtained by rastering the FIB over the sample surface; an area of maximum $500 \times 500 \mu \mathrm{m}^{2}$ can be covered. The more detail on this TOF-SIMS apparatus is described in our previous work [3].

In this study, resonance ionization of uranium was performed as a demonstration experiment of R-SNMS. Details of the sample will be described later. In general, both element selectivity and high ionization efficiency are important features in resonance ionization. Therefore, two- or three-step resonance schemes with visible, or infrared laser wavelengths were used. Since this study aims to introduce Ti:Sa laser into TOF-SIMS apparatus, one color two photons resonance ionization was performed. Figure 3 shows the resonance ionization scheme of uranium using in this study.

Precise time synchronization between the pulsed laser and pulsed FIB was needed. In addition, it was needed that the signals due to secondary ions (generated through non-element selective ionization) were suppressed. For this purpose an appropriate reverse-voltage, as they lead to a disturbing background for the selective RIMS analysis. Two digital delay generators (Stanford Research Systems Inc., DG535) were used for timing adjustment of the FIB, laser, extractor electrode of TOF-mass spectrometer (TOF-Ext) and time-to-digital convertor (TDC). First DG535 was operated with an internal trigger, and the timing of first DG535 was used as a reference of the other triggers. The pump laser was operated with the external trigger from first DG535 included the appropriate delay time. To suppress the sputtered secondary ions, a pulsed counter-voltage (1200 V) is applied to the TOF-Ext, alternating with the extraction voltage $(1600 \mathrm{~V})$ of ions with respect to the TOF-MS entrance. The time sequence of all triggers is shown in Figure 2. Since between the impact of FIB pulse on the sample surface and the post-ionization with the resonant laser is difference, positive secondary ions are pushed back to the sample surface immediately after their release by the counter-voltage on TOF-Ext. After then, the resonant ions are collected and introduce into the TOF-MS by the pulsed extraction field.

\subsection{Sample}

Uranium is a common nuclear material and an object of proliferation. In this study, the autunite was used as 
uranium sputtering target. An autunite is naturally occurring uranium mineral, which chemical formula is $\mathrm{Ca}\left(\mathrm{UO}_{2}\right)_{2}\left(\mathrm{PO}_{4}\right)_{2} \cdot 10-12 \mathrm{H}_{2} \mathrm{O}$. An autunite was acquired as a mineral and its powdery fragment was used as a sample. The autunite particles were pushed onto a smooth indium plate (Nilaco, purity 99.99\%) and mounted on the sample stage. Autunite is a yellow-greenish fluorescent mineral. Before vacuum introduction, the state of the sample was observed with a fluorescence microscope.

\subsection{Ti:Sapphire laser}

The fluctuation of the laser power and instability of wavelength and the optical axis during an R-SNMS measurement affect the count rate of resonantly-ionized ions among a number of pixels in two dimensional elemental distribution image. The repetition rate of the laser influences the analytical time. Therefore, Ti:Sa laser was adopted as high repetition rate and high stability laser for R-SNMS. The Ti:Sa laser used in this study was developed by us base on the conventional Ti:Sa laser [5]. The features of this laser are high repetition rate $(\sim 10 \mathrm{kHz})$, wide tuning range (710 to 940 $\mathrm{nm}$ in fundamental, 360 to $420 \mathrm{~nm}$ in second harmonic), high power $(\sim 60 \mu \mathrm{J} /$ pulse at $800 \mathrm{~nm})$ and high stability. A commercial intracavity doubled $(532 \mathrm{~nm}) \mathrm{Nd}$ :YLF pump laser (Spectra-Physics, Evolution: Model II) with a repetition rate of $1 \mathrm{kHz}$ is applied to simultaneously pump Ti:Sa laser.

\section{Results and discussion}

\subsection{Coupling of TOF-SIMS and RIMS}

For confirmation of R-SNMS, resonance spectrum of uranium was acquired by the following procedure. First, the signal of post-ionized ${ }^{238} \mathrm{U}$ was verified using a rather high power of $50 \mu \mathrm{J} /$ pulse so as to exist the non-resonance ionization. Alignment of the laser optical axis was then performed so that the intensity of post ionized ${ }^{238} \mathrm{U}$ became the maximum with the same power. The wavelength was shifted from the theoretical value and it was lowered to the power of $8 \mu \mathrm{J} /$ pulse, where the

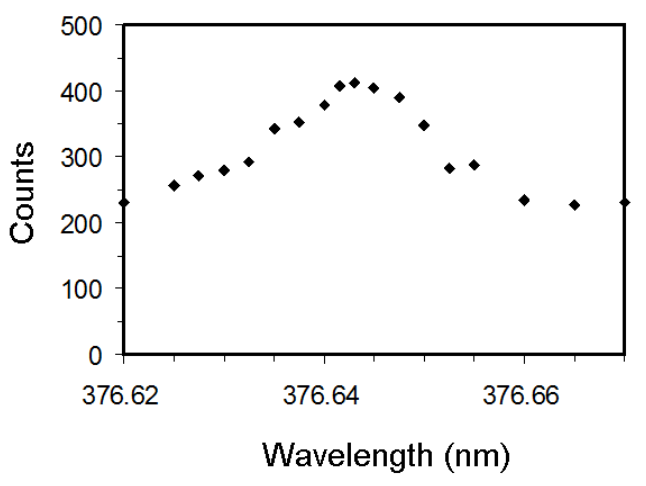

Figure 4. The resonance ionization spectrum of uranium by one color two photons process (at laser power of $8 \mu \mathrm{J} /$ pulse).

non-resonant signal disappeared. Figure 4 shows resonance ionization spectrum of uranium. A signal of resonance ionization was confirmed. The resonance wavelength was $376.642 \mathrm{~nm}$. The coupling between TOF-SIMS and RIMS succeeded. Non-resonance ionized signals and secondary ions that could not be completely removed are included in the background.

\subsection{The R-SNMS imaging of Uranium in autunite}

Figure 5 shows the FIB-induced secondary electron image of autunite and elemental distribution of ${ }^{238} \mathrm{U}$ and $\mathrm{Ca}$ analyzed by TOF-SIMS and R-SNMS. The number of pixels of elemental distribution image analyzed by TOF-SIMS and R-SNMS were $128 \times 128$ pixels. The number of irradiations of the FIB pulse was 60 shots/pixel. The FIB current of $1.3 \mathrm{nA}$ measured in DC mode. The pulse width of the FIB was 200 ns and repetition of $1 \mathrm{kHz}$. The distribution of ${ }^{238} \mathrm{U}$ obtained by R-SNMS was consistent with the results of both SEM and TOF-SIMS. Resonant ionization imaging of uranium was succeeded. However, the intensity of ${ }^{238} \mathrm{U}$ analyzed by R-SNMS was insufficient as compared with the results of TOF-SIMS. The maximum intensity of the pixels in the image of R-SNMS is 3 counts per pixel. As reasons for this, the following can be considered. First, the efficiency of one color resonance ionization process

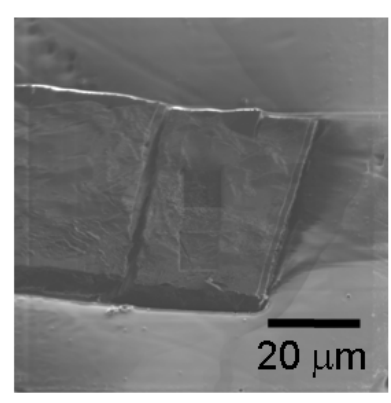

SE image

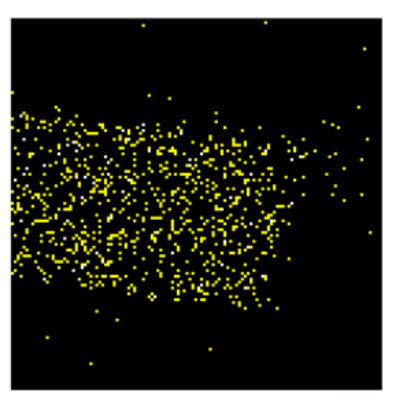

${ }^{238} \mathrm{U}(\mathrm{R}-\mathrm{SNMS})$

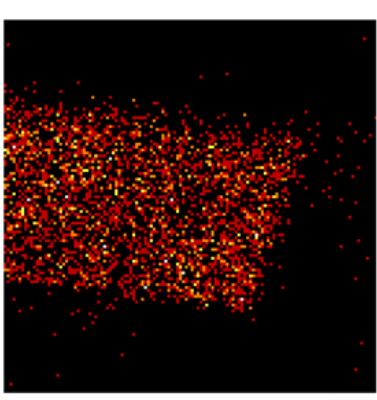

${ }^{238} \mathrm{U}(\mathrm{SIMS})$

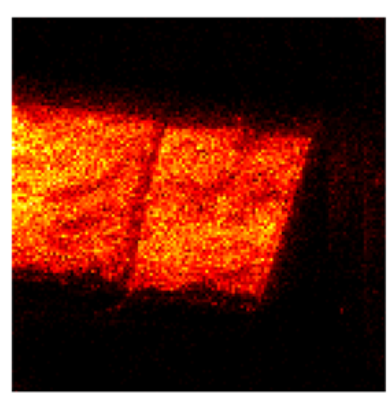

${ }^{40} \mathrm{Ca}$ (SIMS)

Figure 5. The FIB-induced secondary electron image of autunite and the elemental distribution of ${ }^{238} \mathrm{U}$ and $\mathrm{Ca}$ analyzed by TOF-SIMS and R-SNMS. 
is not good compared with two- or three-color process. In addition, since the sputtered neutral particles have a velocity (kinetic energy) distribution and an angular distribution, Doppler broadening may occur. In this situation, not all of the laser axis region can be ionized. Moreover, the number of neutral particles of uranium was small, since the autunite contained a large amount of counter ion such as oxygen. In general, more positive secondary ions are generated as the sample is oxidized. In the future, we will need to add additional lasers and try with a more efficient ionization scheme. Measures against Doppler shift are also necessary.

\section{Conclusion}

The new Ti:Sa laser was developed for R-SNMS was introduced into TOF-SIMS apparatus. The one color two photons resonance ionization of uranium was confirmed. And the micro imaging of resonance ionized ${ }^{238} \mathrm{U}$ from an autunite was obtained. Therefore, micro imaging system for elemental sensitive analysis was developed. In order to increase both selectivity and intensity for target element, and make it a practical apparatus, it is necessary to adopt multi-color resonance ionization method.

\section{Acknowledgements}

This study supported by "Development of System and
Technology for Advanced Measurement and Analysis" SENTAN, JST.

\section{References}

[1] Y. Ranebo, P.M.L. Hedberg, M.J. Whitehouse, K. Ingenerid and $\mathrm{S}$. Littmanna, Improved isotopic SIMS measurements of uranium particles for nuclear safeguard purposes, J. Anal. At. Spectrom. 24 (2009), pp. 277-287.

[2] G. Tamborini and M. Betti, Characterisation of radioactive particles by SIMS, Mikrochim. Acta. 132 (2000), pp. 411-417.

[3] T. Sakamoto, M. Koizumi, J. Kawasaki and J. Yamaguchi, Development of a high lateral resolution TOF-SIMS apparatus for single particle analysis, Appl. Surf. Sci. 255 (2008), pp. 1617-1620.

[4] K. Ohishi, T. Sakamoto, J. Saikawa, N. Ishigaki, K. Tojo, Y. Ido, S. Hayashi, S. Ishiuchi, K. Misawa and M. Fujii, Imaging of polycyclic aromatic hydrocarbons by means of sputtered neutrals mass spectrometry using a diode-pumped solid-state laser, Anal. Sci. 29 (2013), pp. 291-295.

[5] S. Rothe, B.A. Marsh, C. Mattolat, V.N. Fedosseev and K. Wendt, A complementary laser system for ISOLDE RILIS, Journal of Physics: Conference Series 312, (2011), 052020. 